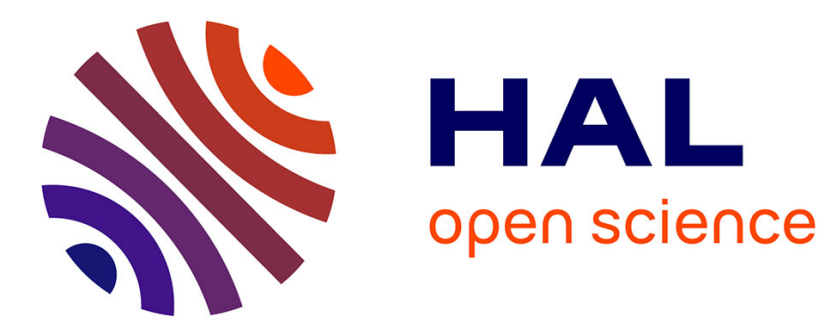

\title{
Des jets géants au-dessus d'un orage isolé proche de la Réunion
}

Serge Soula, Patrice Huet, Oscar van Der Velde, Joan Montanya, Christelle Barthe, József Bór

\section{- To cite this version:}

Serge Soula, Patrice Huet, Oscar van Der Velde, Joan Montanya, Christelle Barthe, et al.. Des jets géants au-dessus d'un orage isolé proche de la Réunion. La Météorologie, 2012, 77, pp.30-40. 10.4267/2042/47373 . hal-01196872

\section{HAL Id: hal-01196872 \\ https://hal.univ-reunion.fr/hal-01196872}

Submitted on 3 Sep 2021

HAL is a multi-disciplinary open access archive for the deposit and dissemination of scientific research documents, whether they are published or not. The documents may come from teaching and research institutions in France or abroad, or from public or private research centers.
L'archive ouverte pluridisciplinaire HAL, est destinée au dépôt et à la diffusion de documents scientifiques de niveau recherche, publiés ou non, émanant des établissements d'enseignement et de recherche français ou étrangers, des laboratoires publics ou privés. 


\title{
Des jets géants au-dessus d'un orage isolé proche de la Réunion
}

\section{Résumé}

Le 7 mars 2010, cinq jets géants ont été enregistrés avec une caméra vidéo noir et blanc et un appareil photo couleur au-dessus d'un orage tropical (température au sommet du nuage $\sim-81^{\circ} \mathrm{C}$ ) à l'est de la Réunion. Grâce à la faible distance d'observation (environ $50 \mathrm{~km}$ ), la lumière produite simultanément dans le nuage et dans le canal des jets géants est analysée en détail pour la première fois. Les sommets des jets géants sont estimés à une altitude comprise entre 80 et $90 \mathrm{~km}$. Les jets géants sont précédés et suivis par des émissions de lumière pulsionnelles au sein du nuage, sans aucun éclair nuage-sol détecté, ce qui suggère qu'ils proviennent principalement de décharges intranuage sans aucun transfert de charge vers la Terre. Selon les rayonnements électromagnétiques enregistrés dans la gamme ELF à Nagycenk (Hongrie), les cinq jets géants transportent des charges négatives au-dessus du nuage. Pendant les jets géants (durée totale de $333 \mathrm{~ms}$ à $850 \mathrm{~ms}$ ), la durée de la phase « leading jet » est celle qui varie le plus (33 à $167 \mathrm{~ms})$. La phase « trailing jet " présente une diminution continue de luminosité dans différentes parties du jet géant et dans le nuage. Le canal inférieur (altitude de 20 à $40 \mathrm{~km}$ ) produit une luminosité bleue qui diminue avec l'altitude et devient de plus en plus diffuse. La région de transition (entre 40 et $65 \mathrm{~km}$ d'altitude) produit une lumière rouge avec des parties plus intenses qui semblent monter lentement $\left(\sim 10^{4} \mathrm{~m} \mathrm{~s}^{-1}\right)$ tout en retraçant les branches initiales de la phase « leading jet ».

\author{
Serge Soula(1), Patrice Huet(2), Oscar van der Velde(3), \\ Joan Montanya( ${ }^{(3)}$, Christelle Barthe ${ }^{(4)}$ et József Bór(5) \\ (1) Laboratoire d'aérologie, université de Toulouse/CNRS, OMP \\ 14 avenue Édouard Belin, 31400 Toulouse \\ (2) La Maison du volcan, muséum de la Fournaise, Bourg-Murat (La Réunion), \\ France \\ (3) Electrical Engineering Department, Technological University of Catalonia, \\ Terrassa, Espagne \\ (4) Laboratoire de l'atmosphère et des cyclones, université de \\ la Réunion/CNRS/Météo-France, Saint-Denis (La Réunion), France \\ (5) Geodetic and Geophysical Research Institute of the Hungarian \\ Academy of Sciences, Sopron, Hongrie
}

\section{Jets bleus et jets géants}

Les jets sont des décharges électriques lumineuses qui se développent audessus d'un nuage d'orage et qui font partie des phénomènes lumineux transitoires (Transient Luminous Events ou TLE, en anglais) regroupant les sylphes, les elves, les jets... (Soula et van der Velde, 2009 ; voir encadré sur les TLE, page 32). Il y a les jets bleus (blue jets, en anglais) qui atteignent 30 à $40 \mathrm{~km}$ d'altitude et les jets géants (GJ ou Gigantic Jets, en anglais) qui atteignent l'ionosphère à environ $90 \mathrm{~km}$ d'altitude, la nuit. Leurs découvertes sont très récentes : 1994 pour les premiers et 2001 pour les seconds. Les jets bleus émergent du sommet du nuage en prenant la forme de cônes de lumière bleutée. Une photo couleur de jet bleu a été prise de l'île de la Réunion par Patrice Huet et publiée par Wescott et al. (2001). Elle est reproduite sur la figure 1a. Les jets géants sont les plus rares et peu de cas ont été documentés depuis leur découverte sur l'île de Porto Rico, qui montrait, pour la première fois, une connexion entre le nuage d'orage et l'ionosphère (Pasko et al., 2002). Dès cette première observation, la vidéo montrait que ce phénomène pouvait être décomposé en deux phases : le « leading jet» qui, dans un premier temps, trace le parcours de la décharge avec des branches plus ou moins développées vers le haut, et le « trailing jet » qui apparaît comme une décharge secondaire, plus lumineuse mais moins développée verticalement (figure 1b). L'année suivante, c'est en Asie, au sud de Taiwan, que l'observation était renouvelée avec, cette fois, cinq cas de jets géants ( $\mathrm{Su}$ et al., 2003). Ces nouveaux cas montraient que la structure verticale n'était pas toujours la même, notamment dans la partie supérieure, avec parfois des petits halos de lumière diffuse (figures $1 \mathrm{c}$ et $1 \mathrm{~d}$ ). $\mathrm{La}$ détection de rayonnements électromagnétiques à extrêmement basse fréquence (ELF ou Extremely Low Frequency, en anglais, c'est-à-dire de 3 à $30 \mathrm{~Hz}$ ), associés à ces premiers jets géants, a permis de déterminer que la charge transportée vers le haut est négative. Une analyse de l'activité d'éclairs nuage-sol (CG ou Cloud-to-Ground, en anglais) d'un orage ayant produit un jet géant dans le sud des États-Unis a montré que ce type de décharge ascendante était probablement très efficace pour décharger le nuage (Van der Velde et al., 2007). La théorie proposée par Krehbiel 


\section{Abstract}

\section{Gigantic jets above an isolated tropical storm near Réunion Island}

On 7 March 2010, five gigantic jets (GJs) were recorded with video and colour cameras above a tropical storm (coldest cloud top temperature $\sim-81^{\circ} \mathrm{C}$ ) east of Réunion Island. Thanks to the small distance of observation $(\sim 50 \mathrm{~km})$, the luminosity within the cloud and within the GJ channels can be analyzed in unprecedented detail. The tops of the GJs are estimated between 80 and $90 \mathrm{~km}$. The GJs are preceded and followed by intermittent optical flashes from the cloud, without any simultaneous cloud-to-ground flash being detected, which suggests they originated mainly as intracloud discharges and without charge transfer to Earth. According to Extremely Low Frequency (ELF) data recorded at Nagycenk (Hungary), the five GJs raise negative charge above the cloud. During the GJ event (333 $\mathrm{ms}$ to 850 ms total duration), the leading jet phase has the most variable duration (33 to $167 \mathrm{~ms})$. The trailing jet phase exhibits a continuous decrease of luminosity in different parts of the GJ and in the cloud. The lower channels ( 20-40 km altitude) produce blue luminosity which decreases with altitude and becomes more and more diffuse with time. The transition region (around 40-65 $\mathrm{km}$ ) consists of bright red luminous beads slowly going up $\left(\sim 10^{4} \mathrm{~m} \mathrm{~s}^{-1}\right)$, retracing the initial leading jet channels.
Figure 2 - Modèle de répartition de charge nuageuse à l'origine du jet bleu (a) et du jet géant (b). (D'après Krehbiel et al., 2008).
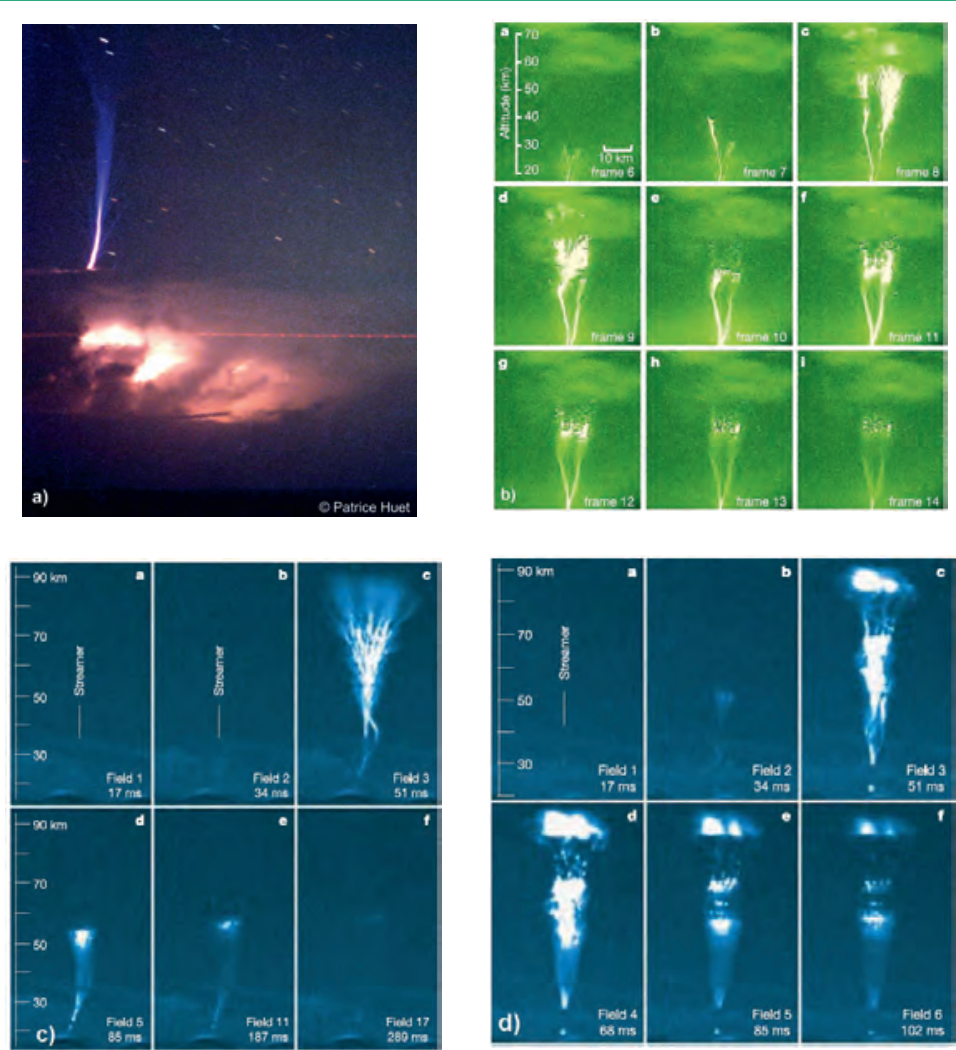

Figure 1 - (a) Photo couleur d'un jet bleu réalisée depuis l'île de la Réunion (@) Patrice Huet). (b) Images successives du premier jet géant vu de l'île de Porto Rico (tiré de Pasko et al., 2002). (c) et (d) Images successives (résolution $\sim 17 \mathrm{~ms}$ ) de deux cas de jet géant vus de Taiwan et dont les structures verticales sont différentes (tiré de Su et al., 2003). Le développement vertical complet persiste plus longtemps dans le deuxième cas.

et al. (2008), et illustrée par la figure 2, explique les différents mécanismes au sein du nuage d'orage qui aboutissent à l'un ou à l'autre des jets. Le jet bleu démarre dans une région de charge positive, au sommet du nuage, tandis que le jet géant commence par une décharge entre une région négative, au cœur du nuage, et une région positive, plus faible, au sommet du nuage. Cette région positive s'épuise avant la fin de la décharge qui continue alors à se propager vers la haute atmosphère. D'une façon générale, ce sont les répartitions et les quantités de charge, dans le nuage et dans son environnement, qui conditionnent le type de décharges qui peuvent se produire.

\section{Les observations du 7 mars 2010}

Le 7 mars 2010, alors qu'un orage s'approche de la côte est de l'île de la Réunion, Patrice Huet, qui travaille à la Maison du volcan et photographie régulièrement les éclairs, aperçoit, d'abord à

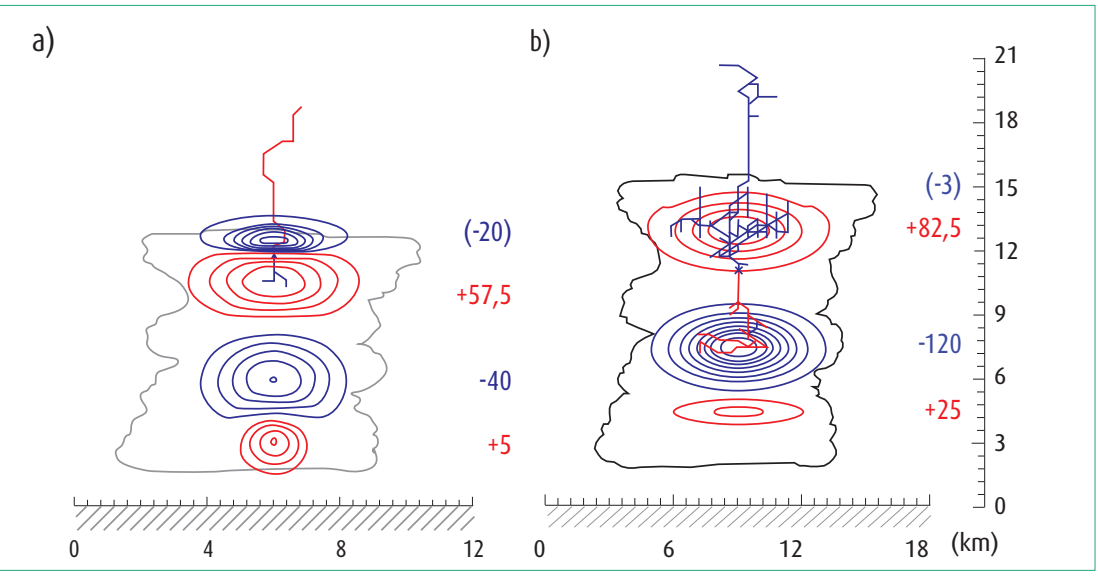




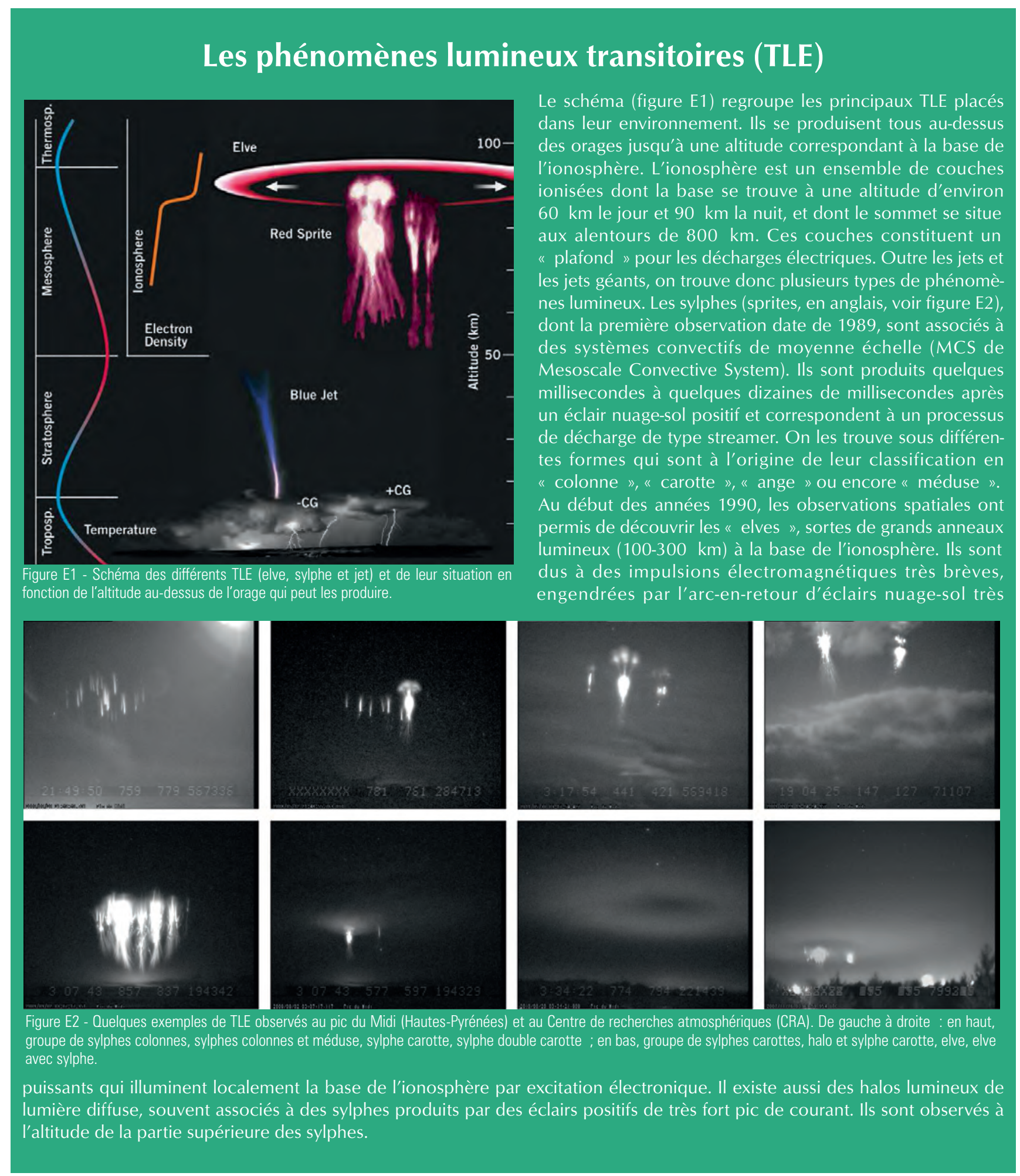

l'œil nu, un phénomène lumineux fugitif au-dessus de la masse nuageuse. Il installe alors son matériel photo et vidéo sur son site d'observation à 1600 mètres d'altitude. Il s'agit d'une caméra vidéo CCD de marque Sony, modèle KPC-350 BH (1/3" ExView HAD CCD), équipée d'un objectif de focale $3,5 \mathrm{~mm}$ et d'ouverture $\mathrm{f} / 1,4$ avec des champs de $70^{\circ}$ horizontalement et $50^{\circ}$ verticalement. Les images de cette caméra ont une résolution temporelle de
16,7 ms entre deux trames successives. L'appareil photo couleur est un NIKON D200 équipé d'un objectif asphérique NIKKOR de focale $18 \mathrm{~mm}$ et d'ouverture $\mathrm{f} / 3,5$. Les clichés réalisés avec cet appareil l'ont été avec une durée d'exposition de 20 secondes et une sensibilité équivalente à 1600 ISO. En moins d'une heure, Patrice Huet obtient, dans ces conditions, cinq enregistrements vidéo de jets géants, à une distance exceptionnellement faible d'environ $50 \mathrm{~km}$, et, pour certains d'entre eux, des photos couleur absolument inédites (Soula et al., 2011).

La figure 3 montre les images extraites des vidéos pour chacun des cinq jets géants dans leur développement vertical complet. Les jets géants ont la forme caractéristique d'un cône inversé audessus d'un tronc plus concentré atteignant 25 à $30 \mathrm{~km}$ d'altitude. L'angle du cône varie de $16^{\circ}$ à $33^{\circ}$, le plus grand 

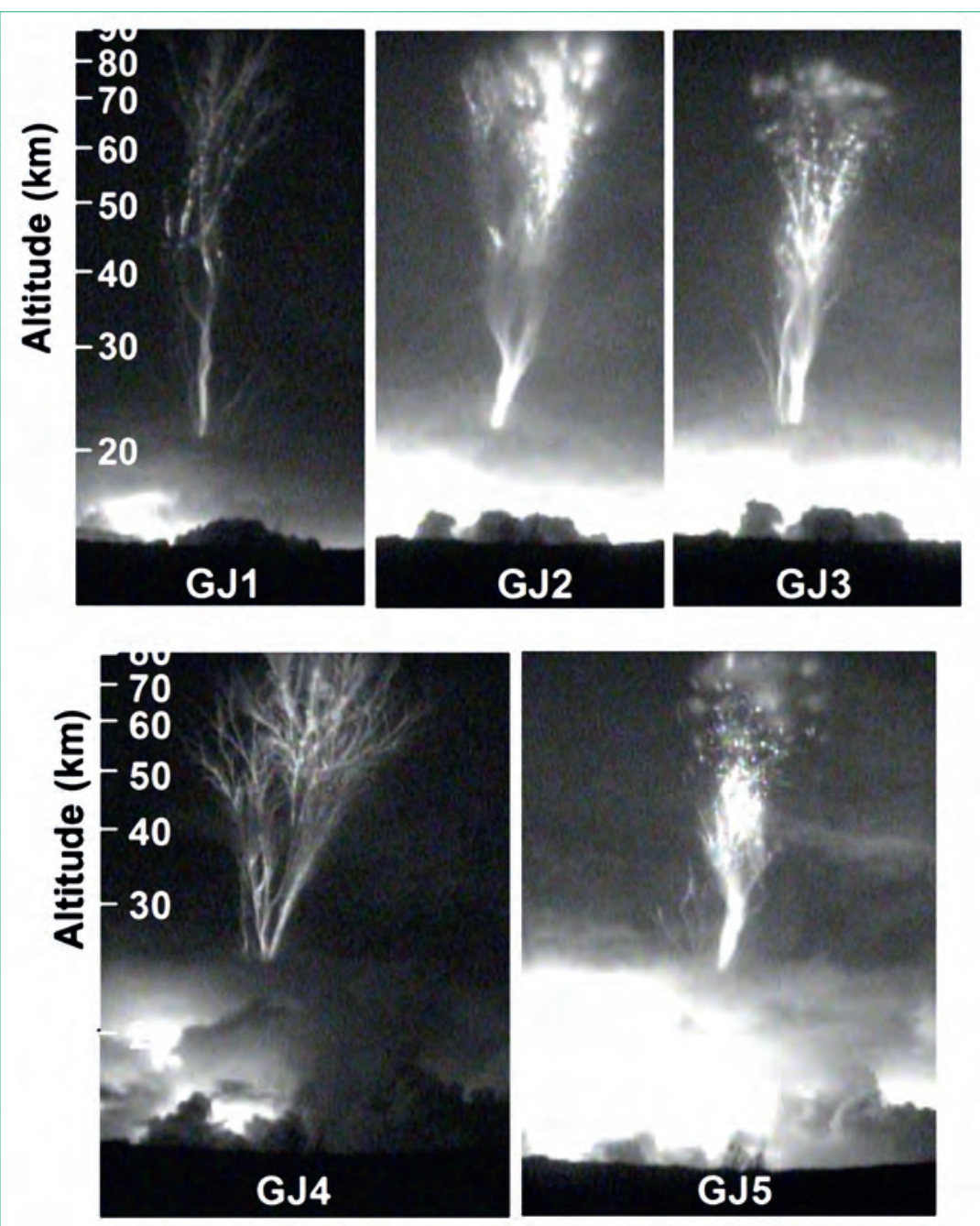

angle étant pour le quatrième jet géant. La distance de l'observateur a été estimée à environ $53 \mathrm{~km}$ pour les trois premiers (GJ1, GJ2 et GJ3) et à $45 \mathrm{~km}$ pour les deux derniers (GJ4 et GJ5).

\section{Caractéristiques générales des cinq jets géants}

Le tableau 1 donne un aperçu des temps d'occurrence des jets géants, de leur durée et de celle de leurs phases principales observées : "leading jet» et " trailing jet » (en distinguant la présence ou non de la région de transition, entre 40 et $65 \mathrm{~km}$, qui s'illumine sous la forme de «perles lumineuses »). Le tableau donne également la durée de l'illumination du nuage qui les produit. Ces durées sont estimées à partir de l'analyse des observations vidéo. D'autre part, le tableau fournit le temps estimé des sources de production ELF et les dimensions verticales des jets géants. Les estimations d'altitude ont une grande incertitude mais le résultat pour les sommets (de 80 à $90 \mathrm{~km}$ ) est similaire aux événements décrits dans la littérature (Pasko et al., 2002 ; Su et al., 2003 ; Van der Velde et al., 2007). La durée totale de la décharge lumineuse visible au sein du nuage varie de 1 à 1,4 seconde alors que la durée de la partie visible du jet géant varie de 0,33
4 Figure 3 - Images tirées des vidéos montrant les cinq jets géants dans leur développement vertical complet. L'échelle verticale prend en compte l'effet de perspective et est seulement valable à la distance du jet géant.

(Tiré en partie de Soula et al., 2011)

à 0,85 seconde. Dans les cinq cas, le jet visible est précédé par de l'illumination continue au sein du nuage avec des impulsions plus intenses.

La figure 4 montre une évolution de la luminosité pour différentes parties du jet GJ5 et pour le nuage. Afin d'optimiser la lisibilité du graphique, le paramètre considéré est le logarithme décimal du nombre de pixels dont la luminosité dépasse un seuil donné. Deux valeurs de ce seuil sont considérées. Les différents graphiques sont fournis en considérant, dans l'imagerie vidéo, la section supérieure du jet géant, sa région de transition, sa partie inférieure et la partie visible du nuage. Le graphe de la luminosité du nuage montre clairement une activité quasi continue pendant tout l'événement dont la durée est de 1,4 s (tableau 1). Toutefois, la luminosité du nuage fluctue beaucoup, avant et après le jet géant, alors qu'elle varie beaucoup plus uniformément au cours des phases du jet géant. Dans ce cas (GJ5), environ sept « pics » de luminosité peuvent être distingués sur une période de $668 \mathrm{~ms}$ avant la partie visible du jet géant. Pour les cinq cas, la durée de cette période initiale d'activité dans le nuage est de 1'ordre de la demi-seconde (300 à $668 \mathrm{~ms}$ ). Le nuage reste lumineux pendant et après la partie visible du jet géant, ce qui suggère un transfert continu de charge. 


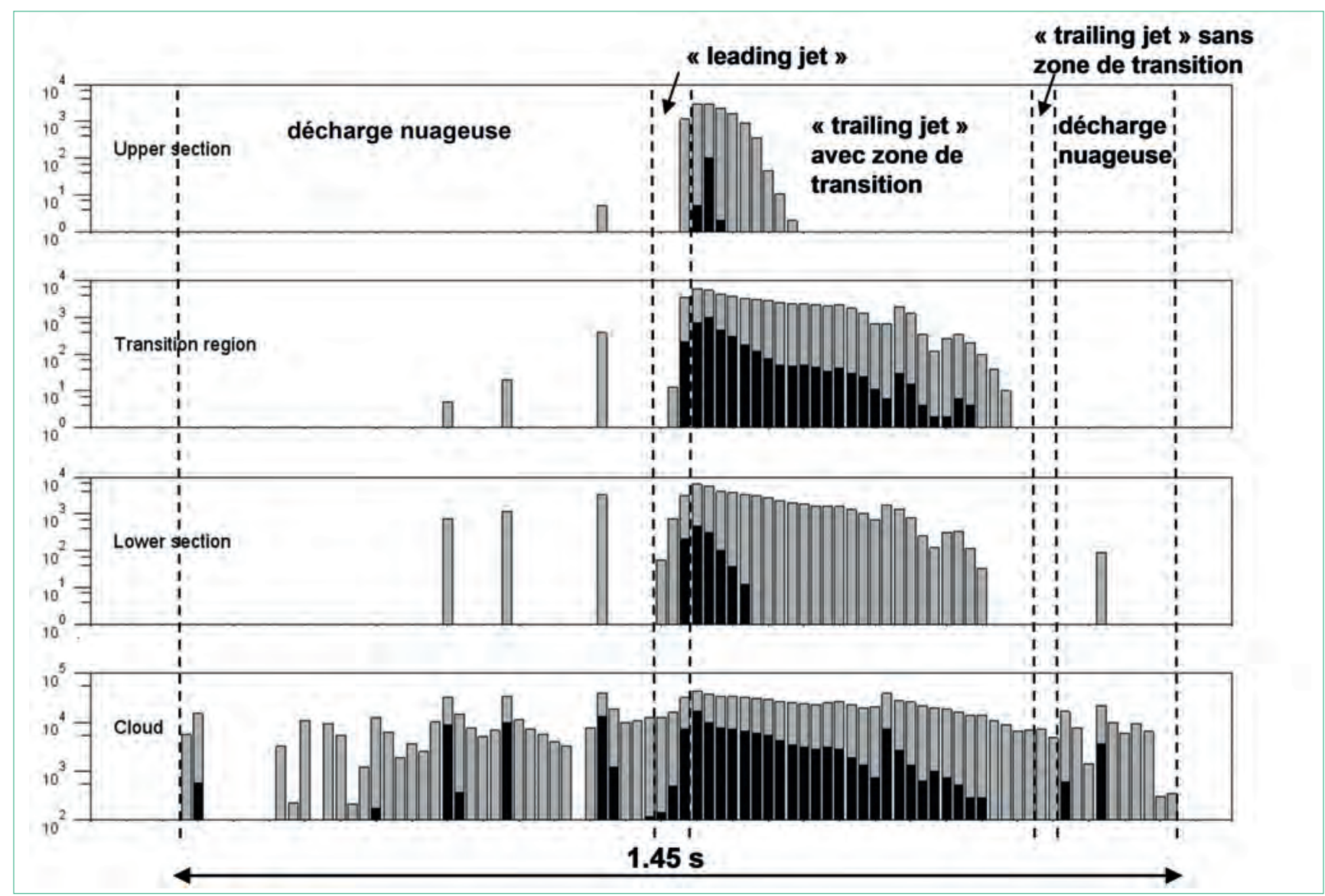

Figure 4 - Variation du logarithme décimal du nombre de pixels de l'image qui dépassent les seuils de luminosité de 10 (gris) et 200 (noir) d'une échelle d'intensité à 8 bits (0-255), au cours de la séquence vidéo correspondant au cinquième jet géant (GJ5). Les quatre graphes correspondent à différentes zones verticales, de bas en haut : nuage (cloud) ; tronc du jet géant de 20 à 40 km (lower section) ; zone de transition de 40 à 65 km (transition region) ; partie supérieure de 65 à 90 km (upper section). (Tiré de Soula et al., 2011 )

\section{Dynamique des cinq jets géants}

\section{Leading jet}

Pour les cinq jets géants, la première phase (ou « leading jet», en anglais) est la plus variable en terme de durée, comme on le voit dans le tableau 1. Elle varie de 33 à $167 \mathrm{~ms}$ (vitesse moyenne de 400 à $2000 \mathrm{~km} \mathrm{~s}^{-1}$ ). Le « leading jet » commence toujours par plusieurs canaux émergeant du haut du nuage, certains d'entre eux atteignant l'altitude du sommet du jet géant $(80-90 \mathrm{~km})$ après des durées variables. Il se termine par une structure très branchue et une luminosité relativement faible, comme le montrent les images de la figure 3 . La figure 5a montre huit des dix images disponibles pour le « leading jet » de l'événement GJ1. Le développement visible vertical de cette phase est estimé à environ $70 \mathrm{~km}$, ce qui signifie une vitesse moyenne de $400 \mathrm{~km} \mathrm{~s}^{-1}$. Les multiples canaux au-dessus du nuage progressent d'abord lentement (environ $100 \mathrm{~km} \mathrm{~s}^{-1}$ ), puis certains accélèrent pour atteindre l'altitude maximale du jet géant. Ainsi la vitesse au début du
Figure 5 - Trames successives, en négatif, tirées des vidéos de trois jets géants. (a) Huit trames pour le premier jet géant pendant sa phase "leading jet " qui dure, dans ce cas, $167 \mathrm{~ms}$. (b) Sept trames pour le troisième jet géant avec la phase " leading jet ", qui dure, dans ce cas, 33 ms (deuxième et troisième trames), et le début de la phase " trailing jet ". (c) Sept trames pour le quatrième jet géant avec la phase " leading jet ", qui dure, dans ce cas, 50 ms (3 premières trames), et le début de la phase " trailing jet ". L'échelle verticale est estimée à la distance du jet géant, dans chaque cas, en prenant en compte l'effet de perspective. (Tiré de Soula et al., 2011)
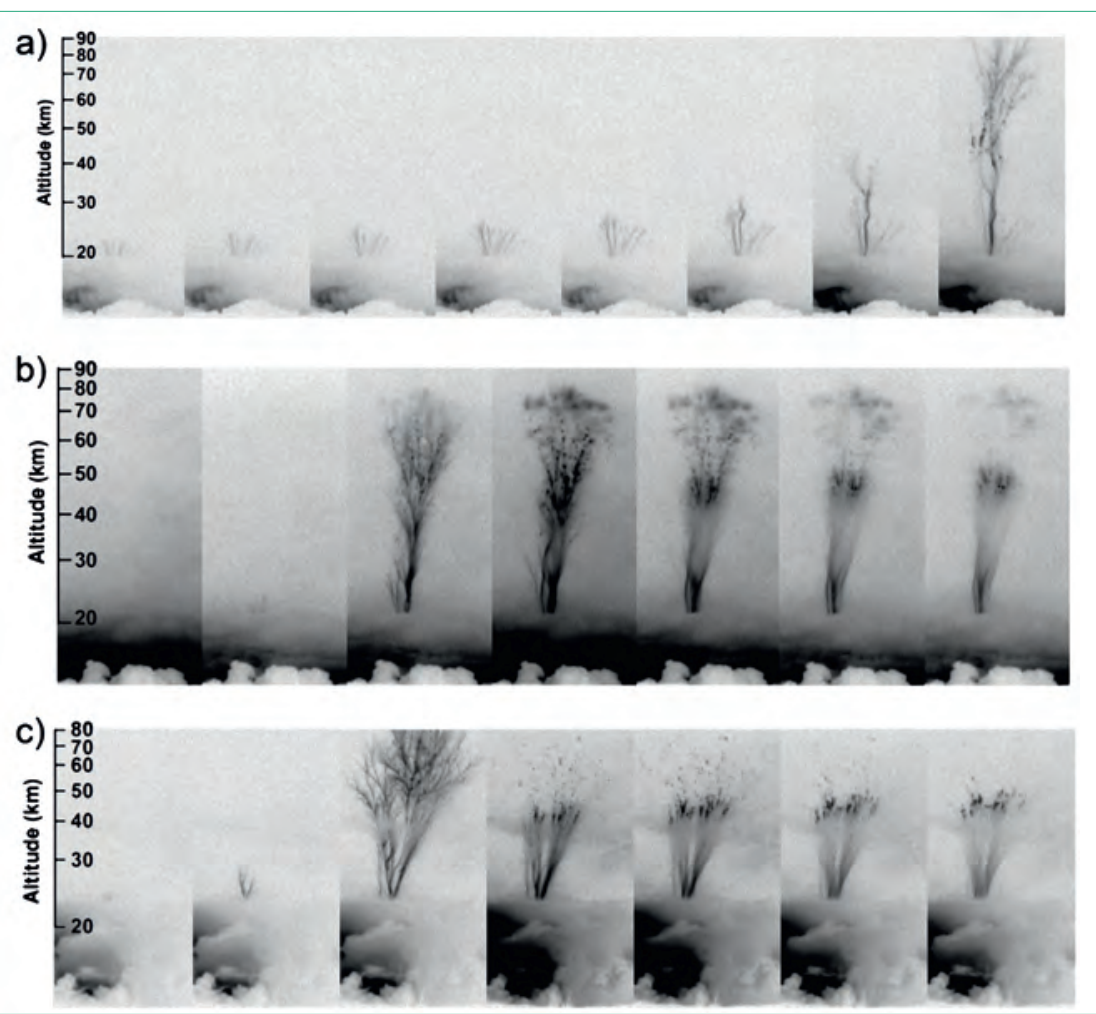
« leading jet » est similaire à celle du traceur d'un éclair nuage-sol (Rakov et Uman, 2003) et peut atteindre des valeurs beaucoup plus grandes (supérieures à $2000 \mathrm{~km} \mathrm{~s}^{-1} \mathrm{~d}$ 'après les images de la figure 5a) dans sa partie supérieure. La figure 5 (b et c) illustre respectivement le développement du « leading jet » le plus bref (événement GJ3) et celui du plus branchu (événement GJ4). Dans ces deux cas, il dure à peine $33 \mathrm{~ms}$ ( 2 images) et $50 \mathrm{~ms}$ ( 3 images), comme l'indique le tableau 1. Dans le deuxième cas, le branchement est très large et dépasse apparemment les limites de l'image. Dans les ramifications, on peut également voir de petits points lumineux qui correspondent à des types différents de décharge électrique.

Dans les deux cas de la figure 5 (b et c), la phase « leading jet » est suivie d'une intensification lumineuse, à la fois dans plusieurs parties du jet géant et dans la partie visible du nuage. Ce stade de l'évolution d'un jet géant évoque une analogie avec l'arc-en-retour de l'éclair nuage-sol qui correspond au maximum d'intensité dans le canal de l'éclair, comme l'ont indiqué Su et al. (2003) ou encore Kuo et al. (2009), en analysant les images de l'instrument ISUAL(1) pour l'observation des TLE depuis l'espace. Pour les deux jets géants de la figure 5 (b et c), lorsque le développement vertical complet est atteint, l'évolution de la structure est assez différente. Dans le premier (GJ3), on note la présence d'un sommet avec une luminosité diffuse et irrégulière qui rappelle la forme d'un sylphe de type « carotte » (Soula et van der Velde, 2009, et encadré). Dans le second (GJ4), cette forme n'est pas visible mais quelques petits points lumineux persistent dans le sommet pendant les quatre images qui suivent le «leading jet ». C'est cette différence d'apparence qui conduit à classer les jets géants dans deux catégories, le type « arbre » et le type « carotte » (Su et al., 2003). Nos images montrent avec beaucoup de détails la structure de cette partie supérieure.

\section{Trailing jet}

Sur la figure 5 (b et c), on voit que la forte luminosité à la fin du développement vertical apparaît à plusieurs niveaux : nuage, base du jet géant, région de transition et, dans un cas, sommet du jet géant. Cette luminosité diminue de façon continue sur les quatre dernières images qui correspondent au début du jet secondaire (ou « trailing jet », en anglais). La figure 6, qui montre cette phase sur une plus longue période pour le cas GJ5, illustre cette diminution. Les 24 images de la figure représentent environ $400 \mathrm{~ms}$ du jet géant visible. Les trois premières images montrent le développement du « leading jet » qui est donc, dans ce cas, de $50 \mathrm{~ms}$. La structure du jet géant correspond ensuite à celle du jet géant GJ3 (type " carotte ») avec un sommet dont la luminosité persiste pendant quelques images. Dès le début du développement vertical complet (quatrième image), la forte luminosité diminue simultanément dans le nuage et dans les trois parties les plus lumineuses du jet géant (le canal principal en dessous de $30 \mathrm{~km}$, la région de transition autour de $50 \mathrm{~km}$ et les patchs de lumière diffuse qui forment la partie supérieure). La figure 4 concerne ce jet géant (GJ5). Elle montre très clairement et de manière quantitative cette diminution de la luminosité sur la période notée « trailing jet », avec région de transition, dont la durée est une demiseconde. La phase « trailing jet » est,

(1) ISUAL : Imager of Sprites and Upper Atmospheric Lightning.

Figure 6 - Trames successives, en négatif, tirées de la vidéo du $5 e$ jet géant (GJ5). Les trois premières trames correspondent à sa phase « leading jet ॥ qui dure, dans ce cas, $50 \mathrm{~ms}$. Les autres trames correspondent à sa phase " trailing jet " qui dure, dans ce cas, près de 0,5 s. L'échelle verticale est estimée à la distance du jet géant en prenant en compte l'effet de perspective. (Tiré en partie de Soula et al., 2011)

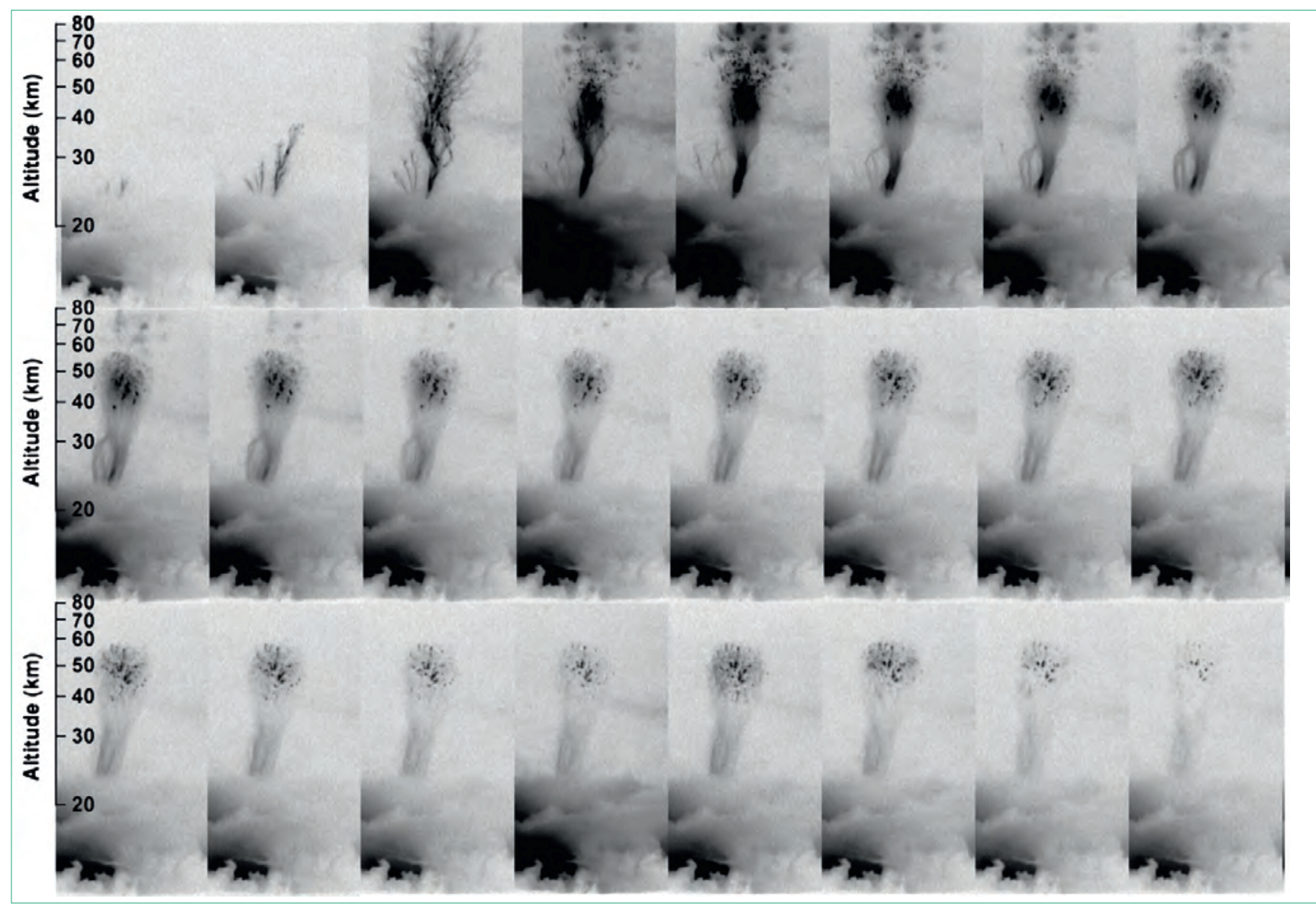


dans tous les cas, celle qui dure le plus longtemps et elle est divisée en deux parties, selon que la région de transition est visible ou pas (figure 4). En effet, la région de transition prend progressivement la forme de « perles lumineuses » qui montent lentement (environ $100 \mathrm{~km} \mathrm{~s}^{-1}$ ) et qui disparaissent avant le canal inférieur du jet géant (figure 6). Cette forme de décharge lumineuse semble indiquer la présence de streamers. Le tableau 1 donne leur durée pour les cinq jets géants observés. La première partie (de $0,20 \mathrm{~s}$ pour GJ3 à 0,47 s pour GJ5) est toujours plus longue que la seconde. Lors de cette diminution de la luminosité, on voit une légère intensification au niveau du nuage (quatrième image de la troisième ligne de la figure 6 et sur le graphe de la figure 4) et au niveau du jet géant dans l'image suivante. Les fluctuations de luminosité, dans le jet géant et dans le nuage, semblent bien être physiquement liées. Cette phase du jet géant rappelle la composante continue de l'éclair nuagesol, à la fois dans la durée, dans la diminution progressive du courant et dans la possibilité d'impulsions de courant appelées composantes M (ou « M-component », en anglais) [Thottappillil et al., 1995]. Dans le cas de la figure 6, on voit également que les branches du « leading jet » n'ayant pas atteint le sommet viennent s'attacher à la branche principale dès le début de la phase « trailing jet».

\section{Les jets géants en couleurs}

La figure 7 montre les photographies en couleurs de deux jets géants : le plus branchu (GJ4) et le plus lumineux (GJ5). Dans chaque cas, la photo est juxtaposée à une image en noir et blanc issue de la vidéo : cinquième image de la figure $5 \mathrm{c}$ pour GJ4 et septième image de la figure 6 pour GJ5. Ce sont les parties les plus lumineuses qui sont visibles sur la photo. Ce qui frappe sur ces premières photos en couleurs de jet géant, c'est le changement de couleur dans la structure verticale du jet géant entre le tronc bleu et la région de transition rouge. Dans le cas de la figure 7a, la région de transition est beaucoup moins visible : seuls quelques filaments lumineux apparaissent. Pour le cas de la figure $7 \mathrm{~b}$, les parties lumineuses sont beaucoup plus visibles, la structure filamenteuse de la région de transition est très nette et la partie diffuse supérieure se devine. Comme pour les sylphes (Soula et van der Velde, 2009) qui sont produits entre $40 \mathrm{~km}$ et $90 \mathrm{~km}$, la

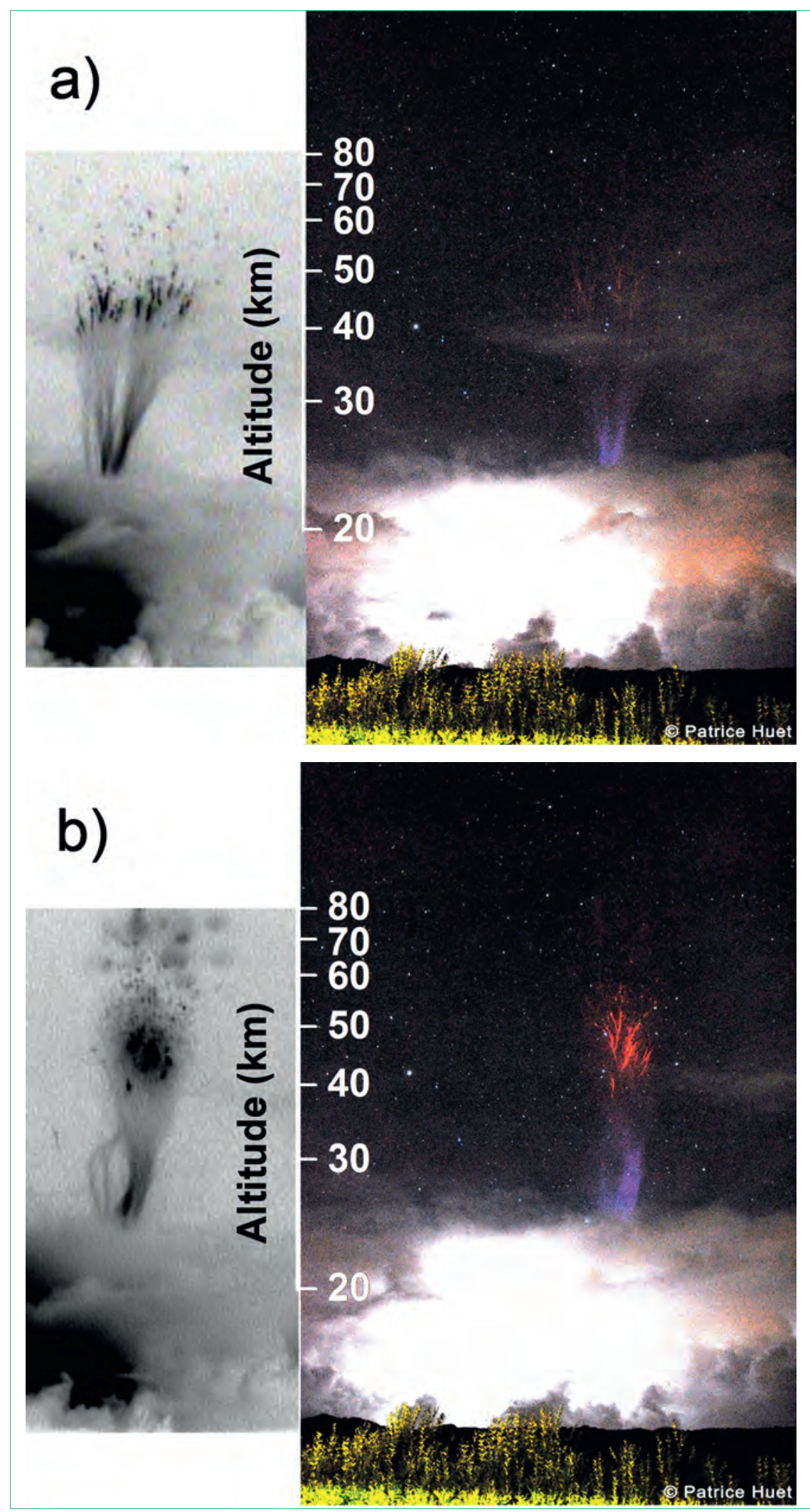

Figure 7 - Image noir et blanc, en négatif, tirée de la vidéo et photo couleur (temps d'exposition 20 s) pour le quatrième jet géant (a) et pour le cinquième jet géant (b). L'échelle verticale est estimée à la distance du jet géant en prenant en compte l'effet de perspective. (Tiré en partie de Soula et al., 2011)

couleur rouge, au-dessus de $40 \mathrm{~km}$, est principalement due à la première bande positive de la molécule d'azote $\mathrm{N}_{2}$ (Pasko et al., 2002). La couleur bleue du canal inférieur est, comme pour les jets bleus, principalement due à la première bande négative de l'ion azote $\left(1 \mathrm{NN}_{2}{ }^{+}\right)$et à la seconde bande positive de la molécule d'azote (2PN2), d'après Wescott et al. (1998). Le bleu de la région inférieure du jet géant présente un aspect diffus qui est essentiellement produit lors de la phase « trailing jet». Le rouge le plus intense de la région de 
transition correspond aux trajectoires de « perles lumineuses » qui retracent les canaux initiaux tracés par le « leading jet ». Cette région produit également une lumière rougeâtre plus diffuse et beaucoup moins intense, tout comme la partie supérieure du jet géant.

\section{Conditions de production des cinq jets géants}

Afin d'analyser les conditions dans lesquelles sont produites ces décharges géantes, nous avons utilisé les températures de sommet de nuage fournies par le radiomètre du satellite Météosat-7, toutes les 30 minutes, et les éclairs détectés par le réseau mondial WWLLN (World Wide Lightning Location Network). Le traitement des données de température prend en compte l'effet de parallaxe pour la localisation en latitude qui se situe entre $20^{\circ} \mathrm{S}$ et $22^{\circ} \mathrm{S}$. Le WWLLN est composé de 40 capteurs répartis dans le monde entier au moment de l'observation et son efficacité de détection sur les arcs d'éclairs nuage-sol est de quelques pour cent dans la région de l'orage (Rodger et al., 2006). Les éclairs sont reconstitués en associant les arcs successifs qui sont à la fois séparés par moins de $10 \mathrm{~km}$ et par moins de $0,5 \mathrm{~s}$.

Les cinq jets géants ont été enregistrés par la caméra à 17 h 40 min $24 \mathrm{~s}$ (GJ1), 17 h $42 \min 49 \mathrm{~s}$ (GJ2), 17 h $44 \min 5 \mathrm{~s}$ (GJ3), 18 h 26 min $6 \mathrm{~s}$ (GJ4), et 18 h 29 min 20 s (GJ5) UTC, le 7 mars
2010. Un peu plus tôt, deux jets géants non enregistrés ont été vus à l'œil nu par Patrice Huet à environ 17 h $34 \mathrm{~min}$ et $17 \mathrm{~h} 36 \mathrm{~min}$ UTC. Ils ont été produits par un orage isolé qui s'est développé au cours de la soirée, à l'est de la Réunion, dans des conditions de convergence d'alizés d'est et nord-est qui ont apporté de l'air chaud et humide, comme souvent sous les tropiques. Le modèle de prévision météorologique globale GFS (Global Forecast System) du service météorologique américain (National Weather Service) indique $65 \mathrm{~kg} \mathrm{~m}^{-2}$ d'eau précipitable (assimilée à la vapeur d'eau intégrée) à $18 \mathrm{~h} \mathrm{UTC}$, une valeur qui n'a que très rarement été trouvée par l'auteur, Oscar van der Velde, à travers les tropiques, dans le même modèle testé sur plusieurs jours. Les valeurs de CAPE (Convective Available Potential Energy ou énergie potentielle de convection disponible) et plusieurs mesures de cisaillement vertical du vent étaient élevées, mais pas de façon extraordinaire.

La figure 8 montre les images de température du sommet des nuages issues de Météosat- 7 dans la région de l'orage $\left(20,5^{\circ} \mathrm{S}\right.$ à $22^{\circ} \mathrm{S}$ en latitude et $55,1^{\circ} \mathrm{E}$ à $57^{\circ} \mathrm{E}$ en longitude) à $17 \mathrm{~h} 38$ et $18 \mathrm{~h} 38$ UTC. Le sommet atteint rapidement $-81{ }^{\circ} \mathrm{C}$ puis s'étale largement. Ces basses températures sont typiques de la tropopause, dans cette région, avec des hauteurs qui peuvent atteindre $18 \mathrm{~km}$ (Sivakumar et al., 2006). La zone avec une température inférieure à $-70{ }^{\circ} \mathrm{C}$ couvre $256 \mathrm{~km}^{2}$ à $17 \mathrm{~h} 38 \mathrm{UTC}$ puis $4900 \mathrm{~km}^{2}$ à $18 \mathrm{~h} 38 \mathrm{UTC}$ et $8000 \mathrm{~km}^{2}$ à 19 h 08 UTC (non montré). Sur la figure 8 , les localisations des coups de foudre détectés par le réseau WWLLN (croix blanches et colorées) montrent la partie occidentale du nuage comme la plus électriquement active. Le triangle indique la position de la station d'observation sur l'île de la Réunion. Les lignes blanches matérialisent l'ouverture du champ de la caméra. Les lignes colorées indiquent les directions des jets géants déterminées grâce aux étoiles en superposant les images à la carte du ciel vu de la station au moment de l'observation. Toutes ces directions passent clairement par la zone la plus active de l'orage.

La figure 9 montre le nombre cumulatif des paramètres d'activité d'éclair (arcs et éclairs issus des données du WWLLN, et éclairs optiques issus des vidéos de $17 \mathrm{~h} 38$ UTC à 18 h 40 UTC), le taux d'éclairs détectés par le WWLLN, les valeurs de la température minimale du sommet des nuages et les occurrences des jets géants. L'activité d'éclair commence à environ $17 \mathrm{~h} 00$ UTC et son taux reste faible (de 1 à 2 par minute) jusqu'à 18 h 00 UTC. Il convient de noter que l'efficacité de détection du WWLLN est beaucoup plus faible que tout autre réseau national de détection et donc ces faibles taux ne sont pas représentatifs des vrais taux mais peuvent indiquer une tendance (Rodger et al., 2006). Les éclairs optiques enregistrés dans les vidéos (courbe rouge) démarrent avec un nombre arbitraire égal à celui des éclairs WWLLN. Les trois premiers jets géants se produisent dans un délai très court (environ quatre minutes), au début de la durée de vie de l'orage, alors que celui-ci n'a pas encore atteint son développement vertical complet et

Figure 8 - Images de la température du sommet de nuage $\left({ }^{\circ} \mathrm{C}\right.$ ) dans la région de l'orage produisant les jets géants : (a) 17 h 38 UTC et (b) 18 h 38 UTC. Les éclairs détectés par le WWLLN, sur une période de 20 minutes centrée sur le temps de l'image Météosat, sont indiqués par des croix blanches (croix rouge et bleu dans (a) pour les arcs associés respectivement à GJ1 et à GJ3). Le triangle indique l'emplacement de la station d'observation. Les directions de visée de GJ1 (bleu), GJ2 (rose), et GJ3 (rouge) sont indiquées en (a), celles de GJ4 et GJ5 (rose) en (b). Les lignes blanches horizontales représentent le champ de la caméra. (Tiré de Soula et al., 2011)

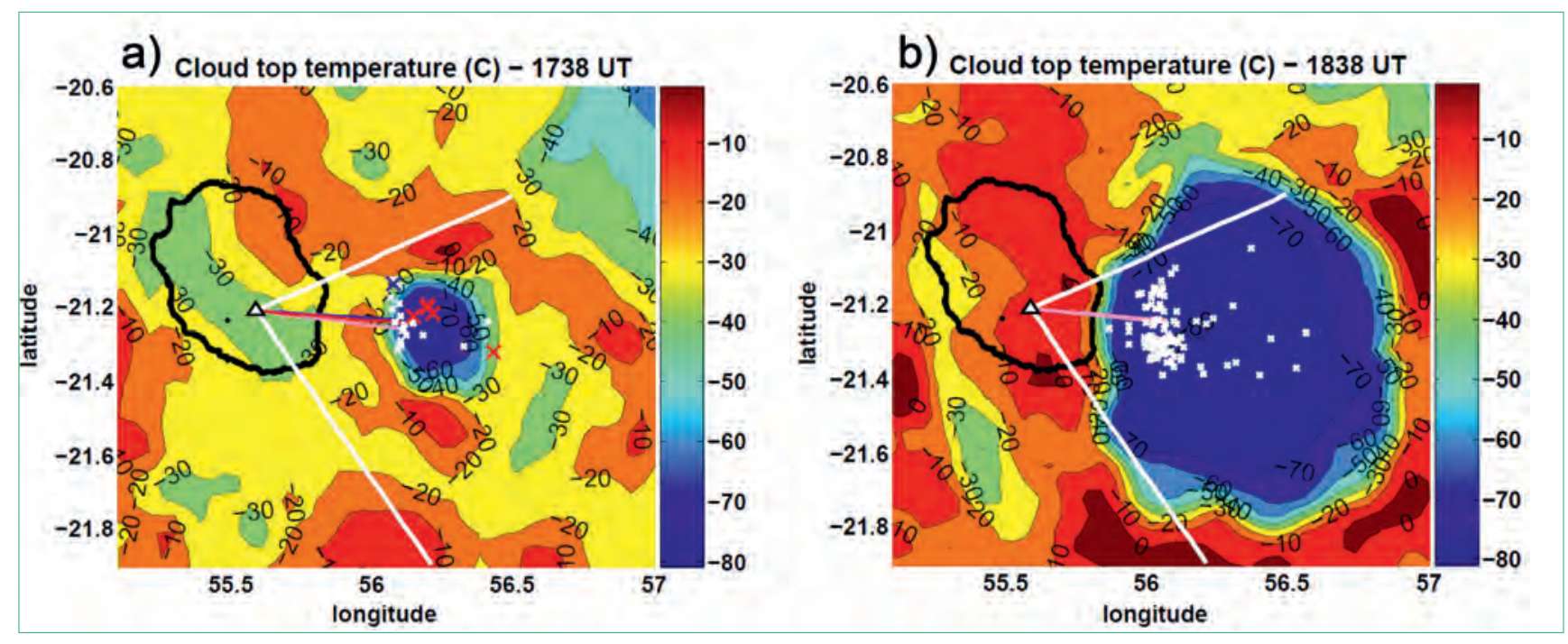




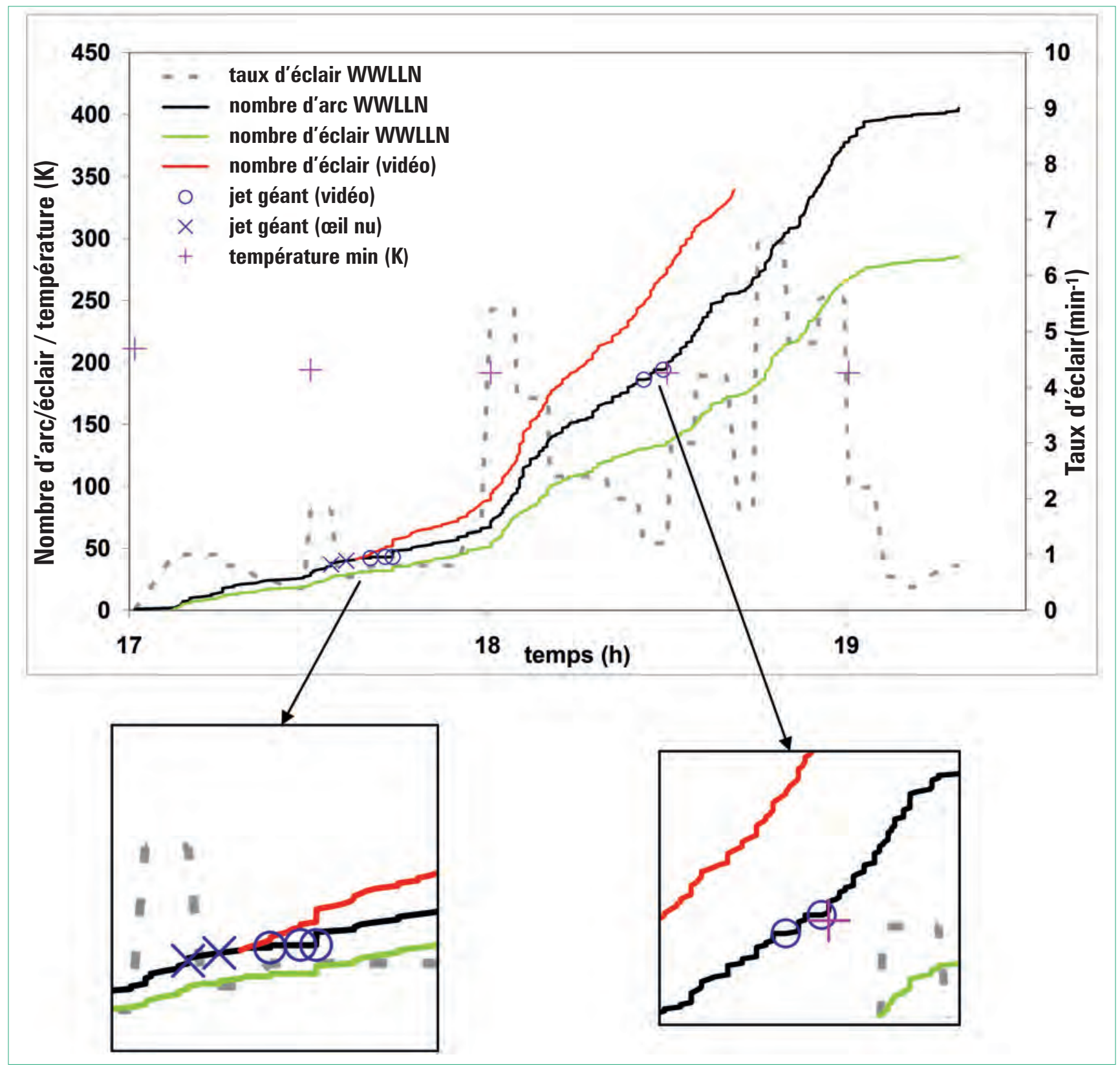

Figure 9 - Évolution de plusieurs paramètres caractéristiques de l'activité d'éclair

- taux d'éclairs détectés par le WWLLN moyenné sur 5 minutes (ligne pointillée)

- nombres cumulatifs des arcs et des éclairs détectés par le WWLLN (traits continus) ;

- nombre cumulatif des éclairs détectés par des méthodes optiques.

Les jets géants observés par vidéo sont indiqués par des cercles et les jets géants observés à l'œil nu sont indiqués par des croix. La valeur de la température minimale du sommet du nuage est indiquée par les croix roses. Au-dessous, se trouvent des zooms sur les deux périodes de production de jets géants. (Tiré de Soula et al., 2011)

avant son expansion horizontale. À partir des images vidéo et de la distance de la partie la plus proche du nuage, l'altitude du sommet des nuages est estimée à 15 $16 \mathrm{~km}$. Pendant un intervalle de $335 \mathrm{~s}$ (de 17 h 38 min $30 \mathrm{~s}$ à 17 h 44 min $5 \mathrm{~s}$ ) précédant le troisième jet géant, un seul éclair a été détecté par le WWLLN environ une demi-seconde après le premier jet géant. Le premier zoom de la figure 9 montre cette période avec un « plateau » pour le nombre d'éclairs détectés par le WWLLN et une augmentation simultanée et continue pour le nombre d'éclairs optiques. Après 18 h 00 UTC, le taux d'éclair est très fluctuant entre 1,2 et 6,6 par minute tandis que les éclairs optiques montrent une augmentation plus prononcée, surtout après $18 \mathrm{~h} 30$ UTC, donc probablement à cause d'éclairs intranuage. Il faut noter que les éclairs détectés par le WWLLN sont essentiellement des éclairs nuage-sol de forte amplitude (Rodger et al., 2006). La zone principale d'activité d'éclairs (figure 8) s'élargit légèrement et se déplace vers l'ouest tandis que la densité d'éclairs augmente nettement. Deux jets géants supplémentaires sont produits vers $18 \mathrm{~h} 30$ UTC alors que le taux d'éclair est relativement faible (1,2 par minute). Le deuxième zoom de la figure 9 montre que chacun de ces deux jets géants est produit sur une période sans aucun éclair nuage-sol détecté par le WWLLN, respectivement de 136 et de 121 secondes, mais avec un certain nombre d'éclairs visibles sur les images vidéo.

\section{Caractéristiques électriques des cinq jets géants}

La datation précise des jets géants nous a permis de rechercher l'existence d'émissions électromagnétiques simultanées. Des signaux dans la gamme ELF correspondant aux cinq jets géants 


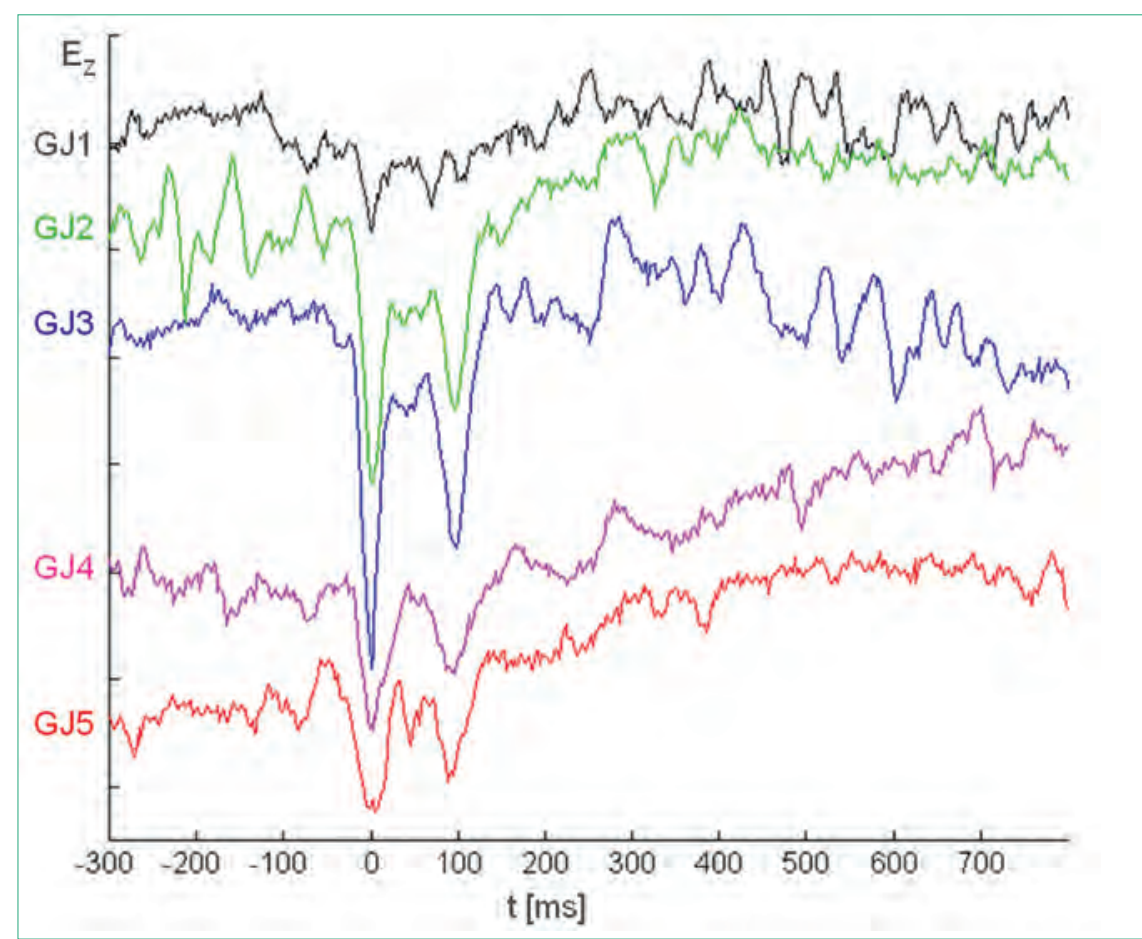

Figure 10 - Série temporelle de la composante verticale du champ électrique $\left(E_{Z}\right)$. Le temps $t=0$ correspond au premier pic du signal pour chaque jet géant. Pour la lisibilité, les amplitudes de chaque cas de jet géant sont décalées sur l'axe vertical et leurs proportions sont respectées. (Tiré de Soula et al., 2011)

ont été détectés à la station de l'Observatoire géophysique près de Nagycenk, en Hongrie $\left(47,62^{\circ} \mathrm{N}\right.$, $\left.16,72^{\circ} \mathrm{E}\right)$. Dans cette gamme de fréquence, les radiations émises par des sources puissantes peuvent se propager sur de grandes distances et l'analyse de l'évolution temporelle des champs électriques et magnétiques associés permet de déterminer la polarité du courant de la décharge (Hobara et al., 2006). Les amplitudes des signaux enregistrés dans notre cas ne sont pas très élevées. Les plus explicites dans les séries temporelles sont ceux de la composante verticale du champ électrique $\left(E_{Z}\right)$ et sont montrés sur la figure 10. Sur ce graphe, le temps $\mathrm{t}=0$ est calé sur le premier pic du signal pour chaque jet géant. Le sens du saut de champ est le même pour tous les événements et il correspond à une charge négative transportée du nuage vers l'ionosphère. Selon la chronologie des vidéos enregistrées à la Réunion et des signaux ELF observés en Hongrie, les jets géants sont de bons candidats pour être à l'origine de ces sauts de champ. Comme le montre le tableau 1, le temps estimé pour la source du signal, en tenant compte de la durée de propagation jusqu'à la station de réception, est très proche de celui du stade de développement maximum du jet géant ( $20 \mathrm{~ms}$ sauf pour GJ4 avec $90 \mathrm{~ms}$ ). Les signaux de la figure 10 correspondant à GJ2, GJ3, GJ4 et GJ5 décrivent un double $\langle\mathrm{V} »$, tandis que celui correspondant à $\mathrm{GJ} 1$ est un triple « $\mathrm{V}$ ». Le deuxième pic est en fait dû à l'arrivée du signal qui s'est propagé autour de la Terre en sens inverse. La durée de crête à crête est pratiquement la même (environ $100 \mathrm{~ms}$ ) pour tous les jets géants. Ces signaux de champ électrique peuvent être utilisés pour estimer la variation du moment de par l'altitude en $\mathrm{km}$ ) produite par la décharge (en ajustant le spectre de la décroissance du signal mesuré à celui d'une décroissance exponentielle) [Huang et al., 1999]. La méthode peut être appliquée à trois des signaux et aboutit à $2800 \mathrm{C} \mathrm{km}$ pour GJ2, à $8100 \mathrm{C} \mathrm{km}$ pour GJ3, et à $1900 \mathrm{C} \mathrm{km}$ pour GJ5, en valeur absolue.

\section{Conclusion}

Cinq jets géants produits par un orage tropical isolé ont été analysés en termes de conditions de production et de caractéristiques lumineuses et électriques. L'orage situé à l'est de la Réunion s'est développé sur la mer et s'est étendu de façon continue pendant deux heures pour couvrir environ $8000 \mathrm{~km}^{2}$. Le sommet du nuage a atteint une température de l'ordre de $-81^{\circ} \mathrm{C}$. La distance de l'observation (environ $50 \mathrm{~km}$ ) a permis l'enregistrement vidéo, en noir et blanc, de la luminosité produite par le nuage et par différentes parties des jets géants, avec des détails sans précédent. De plus, pour la première fois, ont été réalisées des photographies en couleurs charge (produit de la charge en coulomb de ces phénomènes. Les principaux résultats de l'analyse peuvent être résumés de la façon suivante :

- Les jets géants sont regroupés en deux courtes périodes de production, séparées par 40 minutes, et correspondant à deux stades différents du développement orageux. Ces périodes sont caractérisées par un faible taux d'éclairs CG, par rapport à d'autres périodes de la vie de l'orage, et, par ailleurs, l'activité d'éclairs CG a complètement cessé pendant plusieurs dizaines de secondes avant la production des jets géants.

- Les cinq jets géants sont précédés par des émissions lumineuses au sein du nuage, sans aucun éclair CG détecté simultanément, ce qui suggère qu'elles proviennent principalement des décharges intranuageuses, sans aucun transfert de charge vers la Terre. Par ailleurs, les enregistrements de champ électrique rayonné dans la gamme ELF montrent des signatures bien synchronisées avec la production des jets géants, ce qui indique que ces jets géants transportent une charge négative vers l'ionosphère. Les variations de moment de charge induits par les jets géants sont largement supérieures à celles calculées par Cummer (2003) pour les éclairs qui engendrent des sylphes $(\sim 1200 \mathrm{C} \mathrm{km})$, ce qui peut en faire un facteur de décharge du nuage particulièrement efficace. Ces observations confirment la théorie proposée par Krehbiel et al. (2008) qui explique l'émergence du jet géant au sommet du nuage après l'épuisement de la charge positive supérieure, au cours d'une décharge intranuage.

- La phase « leading jet » a la durée la plus variable (33 à $167 \mathrm{~ms}$ ) et devient beaucoup plus rapide au-dessus d'une altitude de $40 \mathrm{~km}$. Toutes les branches de cette première décharge n'atteignent pas le sommet du jet géant.

- Pendant la phase « trailing jet », une diminution continue de la luminosité est observée simultanément dans le nuage et dans différentes parties du jet géant (canal inférieur, région de transition et, pour la plupart des événements, partie supérieure de forme " carotte »). Une possible réintensification lumineuse au cours de cette phase évoque la composante $\mathrm{M}$ du courant continu de certains éclairs nuage-sol.

- Les canaux inférieurs (20-40 km d'altitude) produisent une luminosité bleue qui diminue avec l'altitude et qui devient de plus en plus diffuse. La région de transition (40-65 km d'altitude) se compose d'un rouge vif produit par des décharges lumineuses remontant lentement (environ $100 \mathrm{~km} \mathrm{~s}^{-1}$ ) le long des branches initiales du « leading jet $»$. 
Ces observations de jets géants montrent que les orages, en région tropicale, sont plus favorables à ces phénomènes et nous encouragent à mettre en place des moyens d'observation plus complets pour mieux analyser leur physique et leurs effets sur l'atmosphère. Pour avoir plus de détails sur la physique de ce type de décharge et pour développer sa modélisation, les caméras rapides et la détection de signaux électromagnétiques, dans plusieurs gammes de fréquence, apporteront probablement des informations très intéressantes.

\section{Bibliographie}

- Cummer S. A., 2003 : Current moment in sprite-producing lightning. J. Atmos. Sol. Terr. Phys., 65, 499-508.

Hobara Y., M. Hayakawa, E. Williams, R. Boldi et E. Downes, 2006 : Location and electrical properties of sprite-producing lightning from a single ELF site, in Sprites, Elves and Intense Lightning Discharges. Ed. M. Fullekrug, E. A. Mareev et M. J. Rycroft, NATO Science Series, II. Mathematics, Physics and Chemistry 225, Springer, 398 p.

Huang E., E. Williams, R. Boldi, S. Heckman, W. Lyons, M. Taylor, T. Nelson et C. Wong, 1999 : Criteria for sprites and elves based on Schumann resonance observations. J. Geophys. Res., 104(D14), 16943-16964, doi:10.1029/1999JD900139.

Krehbiel P. R., J. A. Riousset, V. P. Pasko, R. J. Thomas, W. Rison, M. A. Stanley et H. E. Edens, 2008 : Upward electrical discharges from thunderstorms. Nature Geoscience, 1, 233-237, doi:10.1038/nge0162.

Kuo C.-L., J. K. Chou, L. Y. Tsai, A. B. Chen, H. T. Su, R. R. Hsu, S. A. Cummer, H. U. Frey, S. B. Mende, Y. Takahashi et L. C. Lee, 2009 : Discharge processes, electric field, and electron energy in ISUAL-recorded gigantic jets. J. Geophys. Res., 114, A04314, doi:10.1029/2008JA013791.

Pasko V. P., M. A. Stanley, J. D. Mathews, U. S. Inan et T. G. Wood, 2002 : Electrical discharge from a thundercloud top to the lower ionosphere. Nature, 416, 152-154, doi:10.1038/416152.

Rakov V. A. et M. A. Uman, 2003 : Lightning Physics and Effects. Cambridge Univ. Press, Cambridge, U.K.

Rodger C. J., S. Werner, J. B. Brundell, E. H. Lay, N. R. Thomson, R. H. Holzworth et R. L. Dowden, 2006 : Detection efficiency of the VLF World Wide Lightning Location Network (WWLLN): Initial case study. Ann. Geophys., 24, 3197-3214.

Sivakumar V., J.-L. Baray, S. Baldy et H. Bencherif, 2006 : Tropopause characteristics over a southern subtropical site, Reunion Island (21오 $\left.55^{\circ} \mathrm{E}\right)$ : Using radiosondeozonesonde data. J. Geophys. Res., 111, D19111, doi:10.1029/2005JD006430.

Soula S. et 0. van der Velde, 2009 : Phénomènes lumineux transitoires au-dessus des orages : observation et conditions de production. La Météorologie, 8e série, 64, 20-31.

Soula S., O. van der Velde, J. Montanya, P. Huet, C. Barthe et J. Bór, 2011 : Gigantic jets produced by an isolated tropical thunderstorm near Réunion Island. J. Geophys. Res., 116, D19103, doi:10.1029/2010JD015581.

Su H. T., R. R. Hsu, A. B. Chen, Y. C. Wang, W. S. Hsiao, W. C. Lai, L. C. Lee, M. Sato et H. Fukunishi, 2003 : Gigantic jets between a thundercloud and the ionosphere. Nature, 423, 974-976, doi:10.1038/nature01759.

Thottappillil R., J. Goldberg, V. Rakov, M. Uman, R. Fisher et G. Schnetzer, 1995 : Properties of M components from currents measured at triggered lightning channel base. J. Geophys. Res., 100(D12), 25711-25720.

Van der Velde O. A., W. A. Lyons, T. E. Nelson, S. A. Cummer, J. Li et J. Bunnell, 2007 : Analysis of the first gigantic jet recorded over continental North America. J. Geophys. Res., 112, D20104, doi:10.1029/2007JD008575.

Wescott E. M., D. D. Sentman, M. J. Heavner, D. L. Hampton et O. H. Vaughan Jr., 1998 : Blue Jets: Their relationship to lightning and very large hailfall, and physical mechanisms for their production. J. Atmos. Sol. Terr. Phys., 60(7-9), 713-714, doi:10.1016/S1364-6826(98)00018-2.

Wescott E. M., D. D. Sentman, H. C. Stenbaek-Nielsen, P. Huet, M. J. Heavner et D. R. Moudry, 2001 : New evidence for the brightness and ionization of blue starters and blue jets. J. Geophys. Res., 106(A10), 21549-21554, doi:10.1029/2000JA000429 